\title{
Relação entre retinopatia diabética e dermopatia diabética em pacientes portadores de diabetes mellitus tipo 2
}

\author{
Relation between diabetic retinopathy and diabetic \\ dermopathy in type 2 diabetes mellitus patients
}

Hugo Roberto Kurtz Lisboa', Aline Boff', João Rafael de Oliveira Dias ${ }^{3}$, Madalena Rotta ${ }^{4}$, Maiara Garib Guzzo ${ }^{5}$, Saionara Zago ${ }^{6}$, Gláucia Sarturi Tres', Roger Syllos ${ }^{8}$

\section{Resumo}

Objetivos: Dermopatia diabética é o marcador cutâneo mais comum de diabetes mellitus. Embora, a dermopatia e a retinopatia diabéticas sejam consideradas manifestações de microangiopatia diabética, poucos estudos foram publicados a respeito de uma possível associação. Este estudo pretendeu investigar a associação de retinopatia e dermopatia diabéticas e determinar a prevalência e os fatores de risco associados com retinopatia diabética em pacientes diabéticos do tipo 2 do Ambulatório de Diabetes da Faculdade de Medicina da Universidade de Passo Fundo no Hospital de Ensino São Vicente de Paulo. Métodos: Estudo transversal de 90 pacientes diabéticos tipo 2, atendidos sucessivamente em um Ambulatório de Diabetes nos quais foi realizado exame físico dermatológico e oftalmológico. Resultados: A prevalência de dermopatia diabética foi de $16,6 \%(\mathrm{n}=15)$ e a de retinopatia diabética foi de $34,4 \%(n=31)$. Destes, $67,8 \%(n=21)$ consistiam em retinopatia não-proliferativa e $32,2 \%(n=10)$ em retinopatia proliferativa. Observou-se que a duração da doença maior do que 10 anos $(p=0,001)$ e idade maior que 50 anos $(\mathrm{p}=0,014)$ estavam associadas à retinopatia. Não se encontrou associação entre hemoglobina glicada $(p=0,5)$ e glicemia de jejum $(p=0,8)$ com retinopatia diabética. A frequiência de retinopatia diabética em pacientes com dermopatia diabética não foi maior do que nos pacientes sem dermopatia $(7,7 \%, 7$ casos, $p=0,586)$. Não houve associação, estatisticamente significativa, entre retinopatia e dermopatia diabéticas. Conclusão: Não se encontrou associação entre dermopatia e retinopatia diabéticas entre estes indivíduos com diabetes mellitus do tipo 2 . A presença de retinopatia diabética estava associada à duração da doença e à idade avançada dos pacientes.

Descritores: Diabetes mellitus tipo 2/complicações; Complicações do diabetes; Retinopatia diabética/etiologia; Dermatopatias/complicações; Prevalência

'Doutor, Professor de Endocrinologia da Universidade de Passo Fundo (RS), Brasil;

${ }^{2}$ Acadêmica da Faculdade de Medicina da Universidade de Passo Fundo (RS), Brasil;

${ }^{3}$ Acadêmico da Faculdade de Medicina da Universidade de Passo Fundo (RS), Brasil;

${ }^{4}$ Acadêmica da Faculdade de Medicina da Universidade de Passo Fundo (RS), Brasil;

${ }^{5}$ Acadêmica da Faculdade de Medicina da Universidade de Passo Fundo (RS), Brasil;

${ }^{6}$ Mestre, professora de Dermatologia da Universidade de Passo Fundo (RS), Brasil;

Mestre, professora de Clínica Médica da Universidade de Passo Fundo (RS), Brasil;

${ }^{8}$ Oftalmologista, mestre em Farmacologia pela Universidade Estadual de Campinas - UNICAMP - Campinas (SP), Brasil.

Instituição de Realização do Trabalho: Ambulatório de Diabetes da Faculdade de Medicina da Universidade de Passo Fundo e Hospital de Ensino São Vicente de Paulo (RS), Brasil.

Recebido para publicação em: 5/5/2008 - Aceito para publicação em 11/12/2008 


\section{INTRODUÇÃO}

$\mathbf{0}$ diabetes mellitus é uma enfermidade metabólica freqüente caracterizada por hiperglicemia e altera ções no metabolismo de gorduras e proteínas. Este distúrbio é causado pela deficiência relativa ou absoluta de insulina associados ou não à resistência periférica e à ação deste hormônio ${ }^{(1)}$.

É o distúrbio endócrino mais comum, afetando aproximadamente $4 \%$ da população mundial ${ }^{(2)}$. O Diabetes Mellitus tipo 2 (DM2) é a sua forma mais freqüente e a causa é um esgotamento progressivo das células beta das ilhotas de Langherans no pâncreas na tentativa de vencer uma resistência periférica a ação deste hormônio. Sua prevalência vem aumentando, especialmente nos países em desenvolvimento, e estimativas atuais, prevêem o surgimento de mais de 800.000 novos casos por ano ${ }^{(1)}$.

De uma maneira resumida, pode se dizer que a hiperglicemia crônica causa alterações da microcirculação e glicosilação de proteínas, principalmente nas que resultam em lesões que são mais evidentes nos rins, retina, nervos e pele ${ }^{(3)}$. Estes efeitos deletérios na microcirculação se traduzem por insuficiência renal, retinopatia e neuropatia autonômica e periférica e dermopatia diabética ${ }^{(4)}$.

A retinopatia diabética $(\mathrm{RD})$ é a mais grave das várias complicações oculares do diabetes mellitus. Está presente em praticamente todos os portadores de diabetes mellitus com mais de 15 anos de doença. É a principal causa de cegueira em adultos abaixo de 65 anos e a segunda maior causa de novos casos de cegueira na população norte-americana ${ }^{(5)}$. Além disso, pacientes com RD têm risco maior de desenvolvimento de doença coronária, acidente vascular encefálico, nefropatia diabética, amputação de membros e morte ${ }^{(6)}$. Junto à nefropatia e à neuropatia, a RD forma a tríade de complicações microangiopáticas responsável por severa limitação da capacidade funcional dos pacientes.

A presença ou gravidade da retinopatia vai depender de vários fatores, tendo como principais o tipo de diabetes, a duração, o grau de controle metabólico ${ }^{(7)}$, hiperlipemia, gravidez, hipertensão arterial sistêmica ${ }^{(8)}$ e doença renal ${ }^{(9)}$.

Os primeiros sinais clínicos da RD podem ser identificados no exame de fundo de olho pela presença de microaneurismas, exsudatos algodonosos, dilatação capilar, exsudatos duros, microhemorragias puntiformes (nas camadas mais internas da retina) e hemorragias "em chama de vela" (nas camadas superficiais) ${ }^{(10)}$.
A retinopatia diabética pode ser classificada em dois grupos: retinopatia diabética não proliferativa (RDNP) e retinopatia diabética proliferativa (RDP). A RDNP caracteriza-se pela presença de microaneurismas e/ou exsudatos e/ou microhemorragias, na ausência de proliferação neovascular (neovasos). A quantificação destas alterações subdivide este grupo de forma inicial como moderada e grave. RDP é a forma avançada e grave da doença, quando, junto aos achados de forma não proliferativa, observa-se a presença de proliferação neovascular retiniana e/ou vítrea. O principal estímulo desencadeante desta forma mais grave é a hipóxia tissular. A retina isquêmica libera o fator de crescimento do endotélio vascular ou, em inglês, vascular endothelial growth factor (VEGF) e óxido nítrico (NO), aumentando a permeabilidade vascular e estimulando a angiogênese, com conseqüente formação de neovasos, responsáveis pelas mais graves complicações da retinopatia ${ }^{(10)}$.

Manifestações cutâneas do diabetes são bem documentadas, sendo a dermopatia diabética (shin spots) considerada a mais comum. Constitui-se de pápulas ou manchas atróficas pré-tibiais pigmentadas, que delimitam áreas de formato oval ou redondo e distribuem-se bilateralmente, usualmente nos membros inferiores dos diabéticos. É mais freqüente em homens mais velhos, com uma prevalência de $12,5 \%$ a $70 \%$ na população de pacientes diabéticos ${ }^{(11)}$. Um estudo encontrou associação desta lesão a níveis altos de hemoglobina glicada (Alc) ${ }^{(12)}$.As lesões aparecem em áreas cutâneas pobremente vascularizadas e podem servir como um marcador de doença microvascular de outros órgãos ou sistemas ${ }^{(13)}$.

Estudos têm revelado correlação entre dermopatia e outras lesões microangiopáticas do diabetes, como a retinopatia e nefropatia ${ }^{(14)}$.

O objetivo deste estudo foi verificar a presença de associação entre retinopatia e dermopatia diabéticas em pacientes com DM2 do Ambulatório de Diabetes da Faculdade de Medicina da Universidade de Passo Fundo. Concomitantemente verificar os fatores de risco para retinopatia.

\section{MÉTOdos}

Este estudo transversal avaliou 90 pacientes com DM2 atendidos consecutivamente no Ambulatório de Diabetes da Faculdade de Medicina da Universidade de Passo Fundo, localizado no Hospital de Ensino São Vicente de Paulo, município de Passo Fundo, Rio Grande do Sul, 


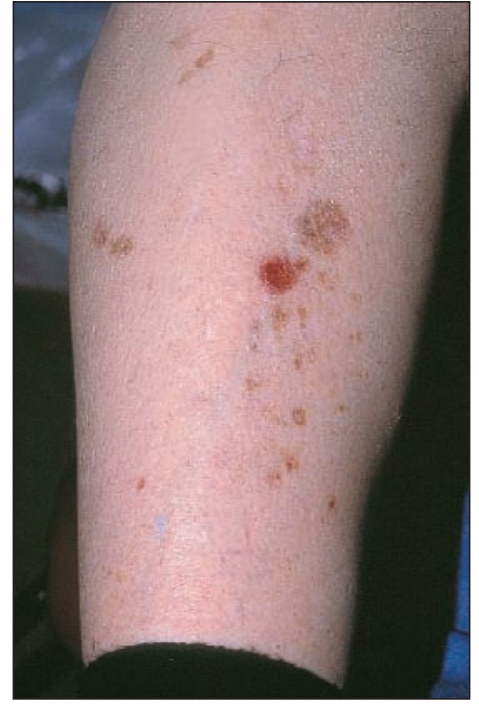

Figura 1: Dermopatia diabética (shin spots)

Brasil, durante um período de seis meses. Foram incluídos pacientes com diagnóstico de DM2, comprovado através de duas medidas de glicemia de jejum maiores ou iguais a $126 \mathrm{mg} / \mathrm{dL}$ ou teste de tolerância oral à glicose com valor maior ou igual a $200 \mathrm{mg} / \mathrm{dL}$, após duas horas da ingestão de 75 gramas de glicose, conforme preconizado pela Organização Mundial da Saúde.

Foram obtidas idade, gênero, duração do diabetes, hemoglobina glicada e glicemia de jejum mais recente. A glicose foi medida pelo método da glicose oxidase e a hemoglobina glicosilada por cromatografia (HPLC) tendo como valores de referência 4,3 a $6 \%$. O exame dermatológico foi feito por médica dermatologista e considerou-se dermopatia diabética a presença de máculas atróficas hiperpigmentadas com a margem distinta, observadas na região pré-tibial ${ }^{(13)}$ (Figura 1). O exame de fundo de olho foi realizado por oftalmologista e a retinopatia diabética foi definida pela presença de lesões não proliferativas ou proliferativas características da doença, através de oftalmoscopia binocular indireta sob midríase ${ }^{(10)}$ (Figura 2).

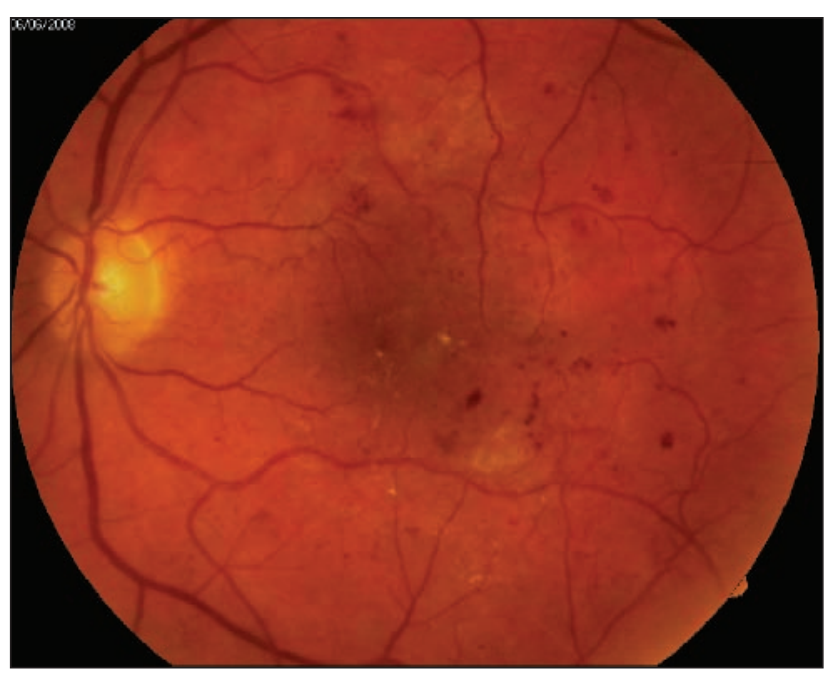

Figura 2: Retinopatia diabética

A análise estatística foi realizada no programa Statistical Package for Social Sciences ${ }^{\mathrm{TM}} 15.0$ (SPSS). Foi obtida a estatística descritiva (média, desvio padrão e mediana). Para variáveis quantitativas foram utilizados os métodos t de Student e análise da variância. Para variáveis qualitativas, foram realizados os testes do quiquadrado e o de regressão logística. Razão de chance (RC) e intervalo de confiança de $95 \%$ (IC 95\%) foram utilizados para mensurar a força de associação entre as variáveis e um valor de $\mathrm{p}<0,05 \%$ foi considerado significante. Todos os pacientes assinaram um termo de consentimento informado, tendo sido o estudo aprovado pelo Comitê de Ética em Pesquisa da Universidade de Passo Fundo.

\section{Resultados}

Entre os 90 pacientes, $50(55,6 \%)$ era do sexo feminino, com idade de 57,27 \pm 9 anos (média \pm desvio padrão) e duração do diabetes de 13,27 \pm 7 anos. Destes $15(16,6 \%)$ apresentaram dermopatia diabética, 31

Tabela 1

\section{Características demográficas do diabetes mellitus tipo 2 com e sem retinopatia diabética}

\begin{tabular}{lccc}
\hline & \multicolumn{2}{c}{ Retinopatia Diabética } & p \\
\cline { 2 - 4 } & Presente $(\mathbf{n}=\mathbf{3 1})$ & Ausente $(\mathbf{n}=\mathbf{5 9})$ & \\
\hline Média de idade \pm DP, anos & $61,77 \pm 8,31$ & $54,9 \pm 9,88$ & $\mathrm{p}=0,001$ \\
Média da duração do & & & \\
diabetes ( $\mathrm{n}=$ 90), em anos & $18,96 \pm 7,03$ & $4,8 \pm 5,91$ & $\mathrm{p}=0,014$ \\
Hemoglobina glicada & $9,13 \pm 1,58$ & $9,43 \pm 1,81$ & $\mathrm{p}=0,5$ \\
Glicemia de jejum & $199,17 \pm 62,21$ & $195,57 \pm 55,82$ & $\mathrm{p}=0,8$ \\
\hline
\end{tabular}


Tabela 2

Resultados de regressão logística de retinopatia diabética de acordo com dermopatia diabética

\begin{tabular}{lcccc}
\hline & Dermopatia & Ausente & Presente & Total \\
\hline Retinopatia & Ausente & 51 & 8 & 59 \\
& Presente & 24 & 7 & 31 \\
Total & & 75 & 15 & 90 \\
\hline
\end{tabular}

$(34,4 \%)$ retinopatia e sete $(7,7 \%)$ apresentaram concomitância dessas duas complicações. Não se encontrou associação entre dermopatia e retinopatia diabéticas $(\mathrm{p}=0,568)$.

Dos pacientes com retinopatia diabética, 17 $(54,8 \%)$ eram do sexo masculino e a média de idade destes foi de $61,8 \pm 8$ anos. Vinte e um pacientes $(67,8 \%)$ apresentavam retinopatia não-proliferativa e $10(32,2 \%)$ retinopatia proliferativa. Vinte e oito pacientes (31\%) tinham mais do que 50 anos e 27 (31\%) evolução do diabetes maior do que 10 anos e observou-se que a duração da doença maior do que 10 anos $(p=0,001)$ e idade maior que 50 anos $(p=0,014)$ estavam associadas à presença de retinopatia

A associação entre retinopatia e os valores mais recentes de hemoglobina glicada e de glicemia de jejum não foi estatisticamente significativa (Tabela 1). Regressão logística mostrou que dermopatia diabética não foi associada com uma maior chance de desenvolvimento de retinopatia diabética (Tabela 2).

\section{Dıscussão}

A prevalência de retinopatia diabética varia na literatura nacional e internacional. Estudo tailandês encontrou prevalência de $31,4 \%$ de retinopatia diabética em 6707 diabéticos do tipo 2, sendo destes 22\% nãoproliferativa e $9,4 \%$ proliferativa ${ }^{(15)}$. A prevalência de retinopatia diabética foi de $24,3 \%$ e $33,9 \%$ nos diabéticos do tipo 2 provenientes de ambulatório de diabetes de Curitiba-PR e Uberlândia-MG ${ }^{(16-17)}$, respectivamente. Encontrou-se uma prevalência de $34,4 \%$ de retinopatia diabética neste estudo, sendo $67,8 \%$ não-proliferativa e $32,2 \%$ proliferativa, valores esses similares a aqueles encontrados nos citados estudos.

O melhor preditor de retinopatia diabética é a duração da doença. Yanko et al. ${ }^{(18)}$ descreveu que a prevalência dessa manifestação após 11 a 13 anos do início da doença foi de $23 \%$, após 16 ou mais anos foi de $60 \%$ e, destes, $3 \%$ tinham retinopatia proliferativa após
11 anos de doença. Vários estudos confirmam a relação entre a presença desta complicação e a duração do diabetes ${ }^{(15,19-20)}$. No presente estudo, também se encontrou associação entre tempo de doença e desenvolvimento de retinopatia, bem como a presença desta complicação em pacientes mais idosos.

São descritos outros fatores de risco para retinopatia diabética, como mau controle glicêmico, presença de microalbuminúria e hipertensão arterial sistêmica. Não se encontrou associação significativa entre os valores elevados de hemoglobina glicada e glicemia de jejum mais recentes com retinopatia diabética, diferindo de outros estudos publicados ${ }^{(1,20)}$. Atribuiu-se este achado à presença de hemoglobina glicada alta nos dois grupos, indicando um mau controle glicêmico dos pacientes. Supõe-se que os indivíduos no grupo sem retinopatia, que eram mais novos, caso não melhorarem seu controle glicêmico virão a apresentar tal complicação. Apesar de o estudo ter correlacionado retinopatia diabética com glicemia de jejum, sabe-se que glicemias capilares em períodos pré e pós-prandiais e, eventualmente reveladas pela monitorização contínua da glicose intersticial, fornecem informações mais precisas sobre as variações da glicemia e podem ser mais fidedignas ${ }^{(21)}$.

Dermopatia diabética usualmente ocorre nas áreas sujeitas a injúria e trauma e são mais comuns nos homens. Embora, não seja encontrada exclusivamente nos pacientes diabéticos, é a manifestação cutânea mais comum dessa doença ${ }^{(22)}$. A freqüência de dermopatia diabética varia na literatura. Neste estudo, dermopatia diabética foi observada em $16,6 \%$ dos pacientes examinados, concordando com estudos previamente publicados. As frequiências encontradas por Sasmaz et al. ${ }^{(23)}$, Romano et al. ${ }^{(24)} \mathrm{e} \mathrm{Lee} \mathrm{et} \mathrm{al.}{ }^{(2)}$ foram 11,$2 ; 12,5$ e $15,5 \%$, respectivamente. Diferentes dados referentes a sexo, duração da doença, idade e o grau da acessibilidade a atendimento médico adequado podem ser alguns dos fatores que levaram à diferença observada entre o presente estudo e os demais citados.

Apesar de retinopatia diabética e dermopatia diabética serem consideradas por alguns autores como sendo manifestações de lesão microangiopática decorrentes da doença, poucos foram os estudos publicados investigando uma possível associação. Nesse estudo não se encontrou relação significativa entre essas duas manifestações. Os resultados são variados na literatura. Um estudo encontrou relação estatisticamente significativa entre ambas, com igual distribuição entre sexos e grupos etários, porém apenas para pacientes com mais de 10 anos de evolução da doença ${ }^{(26)}$. Noutra publicação, os autores não encontraram associação entre essas duas manifestações ${ }^{(24)}$. 
Talvez um número maior de pacientes possam vir a esclarecer mais este assunto e, portanto, mais estudos devam ser realizados para identificar algum marcador cutâneo que possa ser utilizado na identificação precoce de complicações do diabetes mellitus.

Desta forma, continua a recomendação da realização do exame de fundo de olho em todos os pacientes com diabetes do tipo 2 desde seu diagnóstico. Apesar da já estabelecida importância do exame físico e dermatológico para todos os pacientes com diabetes tipo 2, este não poderia ser usado como um marcador para a presença de retinopatia diabética.

\section{Conclusão}

Este estudo não mostrou associação entre dermopatia e retinopatia diabéticas neste grupo de pacientes e, portanto, o achado da lesão cutânea mais freqüente não indica que possa haver a presença de retinopatia.

\section{Abstract}

Purpose: Diabetic dermopathy is the most frequent cutaneous marker of diabetes mellitus. Although diabetic dermopathy and diabetic retinopathy are both considered as manifestations of diabetic microangiopathy, there are only few reports in the literature regarding their possible association. The purpose of this study was to investigate the association between diabetic dermopathy and diabetic retinopathy and to determine the prevalence and the associated risk factors for diabetic retinopathy in type 2 diabetes patients from the Outpatient Diabetic Clinic of the Faculty of Medicine of the University of Passo Fundo and Teaching Hospital Sao Vicente de Paulo. Methods: Cross sectional study was performed in 90 type 2 diabetes mellitus patients who attend to the outpatient diabetic clinic, consecutively. Physical, dermatological and ophthalmologic evaluations were performed in all the patients. Results: The prevalence of diabetic dermopathy was $16,6 \%(n=15)$ and diabetic retinopathy was $34,4 \%$ $(n=31) ; 67,8 \%$ of those $(n=21)$ consisted of nonproliferative diabetic retinopathy and $32,2 \%(n=10)$ of proliferative diabetic retinopathy. The duration of the disease greater than 10 years $(p=0,001)$ and age over 50 years $(p=0,014)$ were associated to retinopathy, but no association was found with elevated levels of $\mathrm{HbAlc}$ $(p=0,5)$ and fasting plasma glucose of 126 or higher ( $p$ $=0,8$ ). The frequency of diabetic retinopathy in patients with diabetic dermopathy was not higher than in patients without dermopathy $(7,7 \%, 7$ cases, $p=0,586)$. There was no statistically significant association between diabetic retinopathy and diabetic dermopathy. Conclusion: No association between diabetic dermopathy and diabetic retinopathy in this group of type 2 diabetes patients was found. The presence of diabetic retinopathy was associated to the duration of the disease and the advanced age of the patients.

Keywords: Diabetes mellitus, type 2/complications; Diabetes complications; Diabetic retinopathy/etiology; Skin diseases/etiology; Prevalence

\section{REFERÊNCIAS}

1. Sherwin SR. Diabetes Melito. In: Goldman L, Ausiello D. Cecil Tratado de Medicina Interna. 22a ed. Rio de Janeiro: Elsevier; 2005. p. $1658-92$

2. King H, Aubert RE, Herman WH. Global burden of diabetes, 1995-2025: prevalence, numerical estimates, and projections. Diabetes Care. 1998;21(9):1414-31.

3. Chakrabarty A, Norman RA, Philips TJ. Feature: cutaneous manifestations of diabetes. Wounds. 2002; 14(8):267-74.

4. Masharani U. Diabetes mellitus and hipoglycemia. In: McPhee SJ, Papadakis MA. Current medical diagnosis \& treatment. 46th ed. Nova York: McGraw-Hill; 2007. p. 1219-1265.

5. Expert Committee on the Diagnosis and Classification of Diabetes Mellitus. Report of the expert committee on the diagnosis and classification of diabetes mellitus. Diabetes Care. 2003;26 Suppl 1:S5-20.

6. Aiello LP, Gardner TW, King GL, Blankenship G, Cavallerano JD, Ferris FL 3rd, Klein R. Diabetic retinopathy. Diabetes Care. 1998;21(1):143-56.

7. Stratton IM, Adler AI, Neil HA, Matthews DR, Manley SE, Cull CA, Hadden D, Turner RC, Holman RR. Association of glycaemia with macrovascular and microvascular complications of type 2 diabetes (UKPDS 35): prospective observational study. BMJ. 2000;321(7258):405-12.

8. Cignarelli M, De Cicco ML, Damato A, Paternostro A, Pagliarini S, Santoro S, et al. High systolic blood pressure increases prevalence and severity of retinopathy in NIDDM patients. Diabetes Care. 1992;15(8):1002-8.

9. Savage S, Estacio RO, Jeffers B, Schrier RW. Urinary albumin excretion as a predictor of diabetic retinopathy, neuropathy, and cardiovascular disease in NIDDM. Diabetes Care. 1996;19(11):1243-8.

10. Grading diabetic retinopathy from stereoscopic color fundus photographs - an extension of the modified Airlie House classification. ETDRS report number 10. Early Treatment Diabetic Retinopathy Study Research Group. Ophthalmology. 1991;98(5 Suppl):786-806.

11. Sibbald RG, Landolt SJ, Toth D. Skin and diabetes. Endocrinol Metab Clin North Am. 1996;25(2):463-72.

12. Jelinek JE. The skin in diabetes. Diabet Med. 1993;10(3):201-13.

13. Perez MI, Kohn SR. Cutaneous manifestations of diabetes mellitus. J Am Acad Dermatol. 1994;30(4):519-31

14. Shemer A, Bergman R, Linn S, Kantor Y, Friedman-Birnbaum R. Diabetic dermopathy and internal complications in diabetes mellitus. Int J Dermatol. 1998;37(2):113-5. 
15. Chetthakul T, Deerochanawong C, Suwanwalaikorn S, Kosachunhanun N, Ngarmukos C, Rawdaree P, et al. Thailand diabetes registry project: prevalence of diabetic retinopathy and associated factors in type 2 diabetes mellitus. J Med Assoc Thai. 2006;89 Suppl 1:S27-36.

16. Ramos SR, Sabbag FP, Busato D, Miranda AB, Moreira Junior CA. Retinopatia diabética: estudo de uma associaçäo de diabéticos. Arq Bras Oftalmol. 1999; 62(6):735-7.

17. Fernandez ML, Moura CA, Jorge PT. Estudo de prevalência de retinopatia diabética no ambulatório da Universidade Federal de Uberlândia. Arq Bras Endocrinol Metab. 1998; 42(5): 368-73.

18. Yanko L, Goldbourt U, Michaelson IC, Shapiro A, Yaari S. Prevalence and 15-year incidence of retinopathy and associated characteristics in middle-aged and elderly diabetic men. Br J Ophthalmol. 1983;67(11):759-65.

19. Casella AM, Bonomo PP, Farah ME. Prevalência da retinopatia diabética em pacientes diabéticos, atendidos pelo Sistema Unico de Saúde (SUS), de Londrina-Paraná. Arq Bras Oftalmol. 1994; 57(1):5-9.

20. González Villalpando ME, González Villalpando C, Arredondo Pérez B, Stern MP. Diabetic retinopathy in Mexico. Prevalence and clinical characteristics. Arch Med Res. 1994;25(3):355-60.

21. Gross JL, Silveiro SP, Camargo JL, Reichelt AJ, Azevedo MJ. Diabetes melito: diagnóstico, classificação e avaliação do controle glicêmico. Arq Bras Endocrinol Metabol. 2002; 46(1):16-26
22. Weismann IK. Skin disorders in diabetes mellitus. In: Burns T, Breathnach S, Cox N, Griffiths C, editors. Rook's textbook of dermatology. 7th ed. Oxford: Blackwell Science; 2004. p.106-9.

23. Sasmaz S, Buyukbese MA, Cetinkaya A, Celik M, Arican O. The prevalence of skin disorders in type- 2 diabetic patients. Internet J Dermatol [Internet]. 2005 [cited 2008 Aug 22]; 3(1). Available from: http://www.ispub.com/ostia/ index.php?xmlFilePath=journals/ijd/vol3n1/diabetes.xml

24. Romano G, Moretti G, Di Benedetto A, Giofrè C, Di Cesare E, Russo G, Califano L, Cucinotta D. Skin lesions in diabetes mellitus: prevalence and clinical correlations. Diabetes Res Clin Pract. 1998;39(2):101-6.

25. Lee $\mathrm{CH}$, Tey BH, Chew W. Epidemiology of diabetes mellitus in a regional hospital medical unit. Ann Acad Med Singapore. 1993;22(6):864-9.

26. Abdollahi A, Daneshpazhooh M, Amirchaghmaghi E, Sheikhi S, Eshrati B, Bastanhagh MH. Dermopathy and retinopathy in diabetes: is there an association? Dermatology. 2007;214(2):133-6.
ENDEREÇO PARA CORRESPONDÊNCIA:
Hugo Roberto Kurtz Lisboa
Rua Teixeira Soares, $n^{\circ}$ 885/806
CEP 99010-901 - Passo Fundo - RS
Tel: (54) 33116499
E-mail: hlisboa@via-rs.net 